\section{Liver transplantation for metastatic neuroendocrine tumors}

Louise Barbier', Cindy Neuzillet ${ }^{2}$, Safi Dokmak', Alain Sauvanet', ${ }^{1}$ Philippe

Ruszniewski \& Jacques Belghiti ${ }^{* 1}$

\section{Practice points}

When is liver transplantation considered as a valuable option in metastatic neuroendocrine tumor patients?

- Two main indications: uncontrolled liver metastases and life-threatening hormonal disturbances.

What are the contraindications to liver transplantation?

- Most prevalent: Ki67 index $>20 \%$, extrahepatic disease, poor performance status.

- Relative: Ki67 >10\%, unknown primary tumor, primary tumor without portal vein drainage, hepatomegaly or major tumor bulk, hepatic or hilar lymph node involvement, upper abdominal exenteration with multivisceral transplantation.

How should the staging of the disease be performed?

- Extrahepatic disease should be ruled out by appropriate morphological assessment, including somatostatin receptor imaging.

- Ki67 should be assessed in both primary and metastatic sites.

What is the strategy with the primary tumor?

- The primary tumor should be resected before indicating liver transplantation (LT).

- Primary tumor should preferentially be located in the digestive system with portal venous drainage (midgut).

- Unknown primary is not a formerly contraindication to LT

What are the results in terms of operative mortality?

- Post-LT mortality varied from 5 to $14 \%$ in large series.

- Major concomitant resections, particularly pancreatic resections, increase mortality after LT.

What are the results of long-term survival?

- Five-year overall survival rates varied from 50 to $80 \%$, depending on indications of LT.

SUMMARY Neuroendocrine tumors are a heterogeneous group of rare epithelial tumors. Most of them are metastatic at the time of initial diagnosis. Liver transplantation (LT) has been proposed in selected patients with diffuse liver involvement. Rationale for LT in this indication is based on the frequently indolent nature of neuroendocrine tumors, the propensity for liver-only metastasis and the high rate of intrahepatic recurrence after resection. However, indications for LT for neuroendocrine liver metastases remain controversial, and patient selection criteria is still a matter of debate. The aim of this review is to summarize data regarding LT for neuroendocrine liver metastases by answering frequently asked questions about this issue.

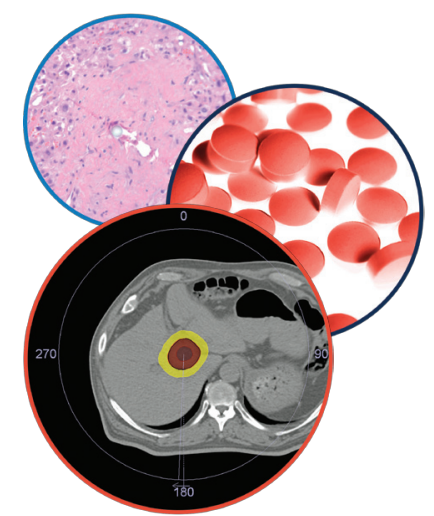

Hepatic Oncology
KEYWORDS

- carcinoid tumor

- Ki-67 antigen • liver transplantation

- neuroendocrine tumor - survival

'Department of HPB Surgery \& Liver Transplantation, Beaujon Hospital (Assistance Publique Hôpitaux de Paris), University Denis Diderot Paris 7, 100 Bd du Général Leclerc, 92110 Clichy, France

${ }^{2}$ Department of Oncology, Beaujon Hospital (Assistance Publique Hôpitaux de Paris), University Denis Diderot Paris 7, 100 Bd du Général Leclerc, 92110 Clichy, France

${ }^{3}$ Department of Gastroenterology, Beaujon Hospital (Assistance Publique Hôpitaux de Paris), University Denis Diderot Paris 7, 100 Bd du Général Leclerc, 92110 Clichy, France

*Author for correspondence: Tel.: +33 1408758 95; Fax: +33 14087 17 24; jacques.belghiti@bjn.aphp.fr

Future $\%$ isg 
Neuroendocrine tumors (NETs) are considered rare malignancies, although their incidence and prevalence have significantly increased over the last two decades [1]. They represent a heterogeneous group of epithelial neoplasms that can arise from neuroendocrine cells that are scattered throughout most organs, including the lung, pancreas and digestive tract (Table 1).

As for many neoplasms, the development of liver metastases (LMs) represents a turning point in the course of the neuroendocrine disease and is a major prognostic factor. Moreover, digestive NETs are frequently metastatic at the time of diagnosis [2]. In specialized centers, $80-90 \%$ of patients with midgut NETs and $60-70 \%$ of those with pancreatic NETs present with distant metastases at diagnosis [2]. However, in contrast to other malignancies, metastatic NETs may be indolent, with prolonged stable disease or slow progression. The evolution of neuroendocrine LMs is dependent on primary tumor site, tumor extent, histological differentiation, proliferative activity and functional status. In addition, progressive LMs, liver tumor burden, presence of extrahepatic disease and carcinoid heart disease also negatively influence prognosis and should be taken into account in the therapeutic decision.

Neuroendocrine LMs are a rare indication for liver transplantation (LT). NETs are noticeably almost the only indication for LT in a malignant disease setting, with the exception of hepatocellular carcinoma. Rational for LT in welldifferentiated NETs is based on the frequently indolent nature of NETs, the propensity for liver only metastasis and the high rate of intrahepatic recurrence after resection. In Europe, NETs account for $0.4 \%$ of total indications for LT since 1988 [3]. Several factors contribute to the rarity of LT in case of metastatic NETs: the high recurrence rate of neuroendocrine disease after LT, the scarcity of liver grafts and the slow evolution in some cases that might not require such aggressive treatment.
Mostly case reports and small series have been published since the 1990s. The publication of larger cohorts of patients [4-9], including our experience (Tables $2 \& 3$ ) contributed to the better knowledge of prognostic factors of survival after LT for neuroendocrine LMs. However, LT for neuroendocrine LMs remains controversial, and patients' selection criteria are still a matter of debate. The aim of this review is to summarize data regarding LT for neuroendocrine LMs by answering frequently asked questions about this issue.

\section{How are NETs classified?}

NETs are classified on the basis of their site of primary origin, histological differentiation (well-differentiated/NETs vs poorly-differentiated/carcinomas) and proliferative activity or grading (G1-G3) (Table 1, WHO Classification of Tumours of the Digestive System, 2010 [10]), as well as their functional status, which is based on the presence of a clinical hormonal syndrome caused by excessive hormone secretion by cancer cells (30-40\% of digestive NETs) [21]. The most common one is the carcinoid syndrome, which is characterized by flushing and diarrhea, and caused by the release of serotonin by lung or midgut tumors with low proliferative activity (most of them are G1). Digestive NETs can produce other peptides, accounting for a broad variety of clinical and biological hormonal syndromes. Insulinomas and gastrinomas are the most frequent functional pancreatic NETs [22].

Histological differentiation, proliferative activity and presence of metastatic disease are the strongest prognostic factors.

What are the therapeutic options when LMs from NETs are discovered?

Frilling et al. for the Working Group on Neuroendocrine Liver Metastases and Pavel et al. proposed recommendations for the treatment approach to neuroendocrine LMs $[2,23]$. As described by Pavel et al., therapeutic

Table 1. WHO classification and grading (2010) of gastro-entero-pancreatic neuroendocrine neoplasms.

\begin{tabular}{|c|c|c|c|c|c|}
\hline & & & & Mitotic count & Ki67 index \\
\hline \multirow[t]{2}{*}{ Neuroendocrine tumor } & Low grade & G1 & & $<2$ mitoses/hpf & $\leq 2 \%$ \\
\hline & Intermediate grade & G2 & & 2-20 mitoses/hpf & $3-20 \%$ \\
\hline $\begin{array}{l}\text { Neuroendocrine } \\
\text { carcinoma }\end{array}$ & High grade & G3 & $\begin{array}{l}\text { Small cells or } \\
\text { large cells }\end{array}$ & $>20$ mitoses/hpf & $>20 \%$ \\
\hline
\end{tabular}


Table 2. Indications of liver transplantation for neuroendocrine tumors liver metastases (series of at least 10 patients, published in English after 1990).

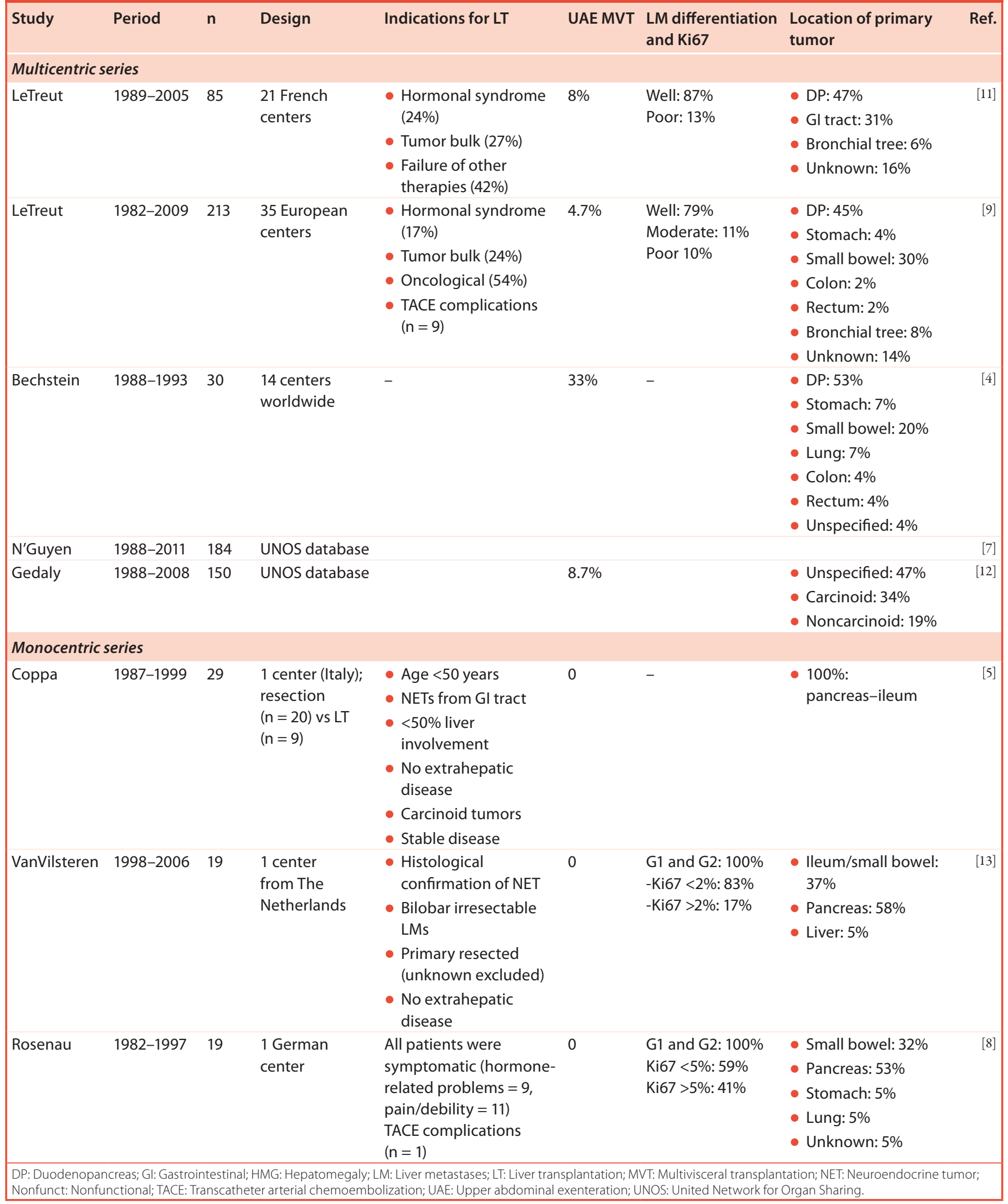


Table 2. Indications of liver transplantation for neuroendocrine tumors liver metastases (series of at least 10 patients, published in English after 1990). (cont.)

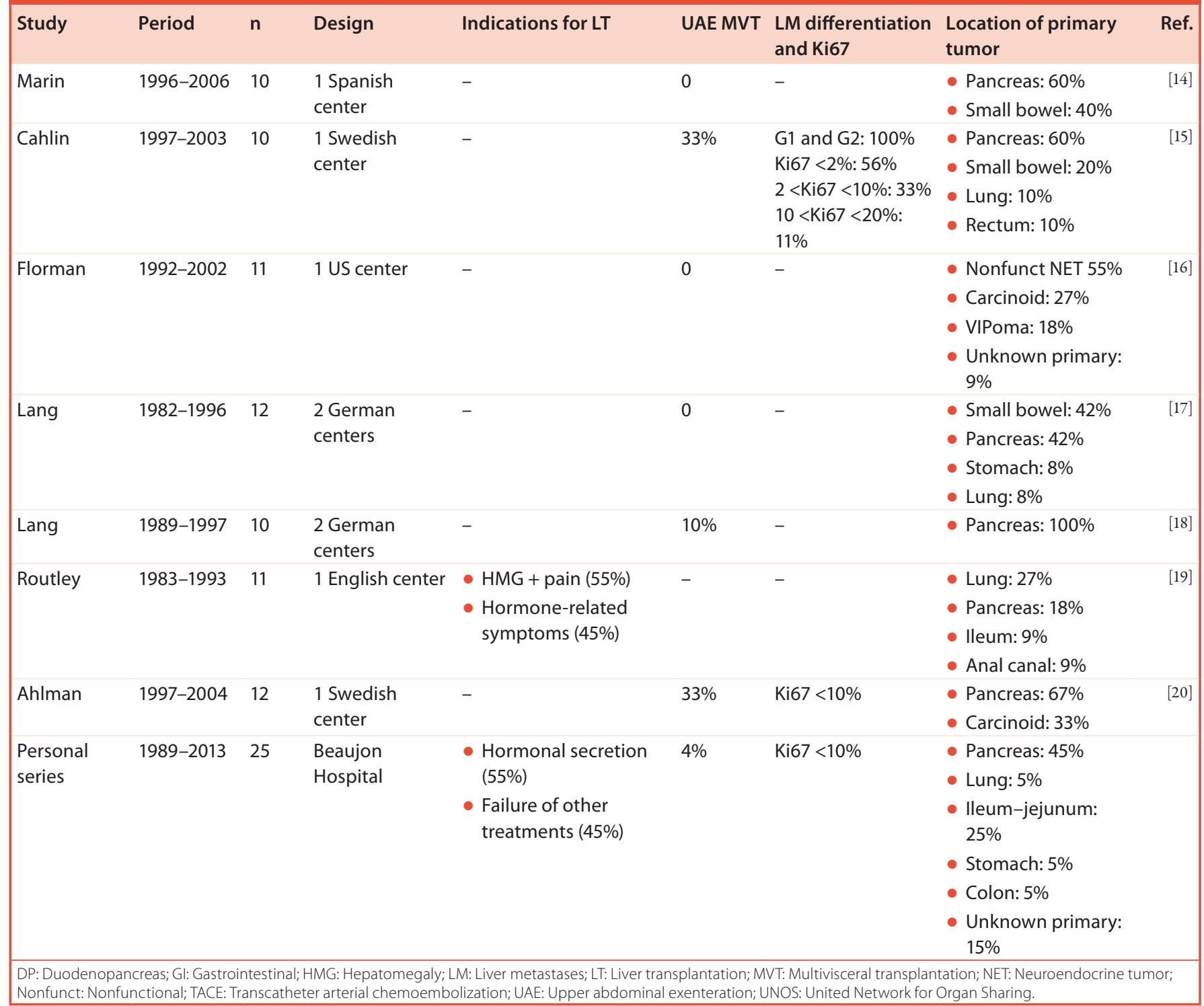

options that can be proposed for patient with NET LMs without extrahepatic spread are the following [2]:

- Patients with simple (unilobar or limited LMs, pattern A) or complex (bilobar LMs, pattern B) LMs of G1/G2 NETs, in the absence of progression in tumor follow-up before surgery, are candidates for surgical resection with or without local ablative techniques. However, although liver-directed surgery for neuroendocrine LMS is the only potentially curative option and is associated with prolonged survival (with 5-year survival rates ranging from 60 to $80 \%$ in recent series), recurrence is near universal, with $>90 \%$ of patients recurring by 5 years, most of them within the remnant liver $[24,25]$;

- Patients with diffuse LMs (pattern C) or $\mathrm{Ki} 67>10-15 \%$, and those who are poor surgical candidates may be treated with somatostatin analogs (SSA), chemotherapy, molecular targeted therapy (sunitinib or everolimus), peptide receptor targeted radiotherapy or transcatheter arterial chemoembolization (TACE), depending on primary tumor site and individual conditions. In highly selected patients with pattern C LMs $(<1 \%)$, LT is presented as an option. 
What were indications for LT in large series of NET patients?

First, LT was performed in NET patients with life-threatening hormonal disturbances refractory to surgical or any other therapy $[8,9,11,19,26,27]$.

Second, symptoms related to major tumor bulk, such as pain or debility, were considered as indications for LT $[7,8,24,26]$. In these cases, the median weight of the explanted liver often exceeded $3 \mathrm{~kg}[7,8]$. In the European series published in 2013 [9], more than half of the patients presented hepatomegaly (HMG) at the time of LT: although the exact extent of liver involvement was not available, HMG was most probably associated with a metastatic disease occupying more than $50 \%$ of the liver. In multivariate analysis, HMG was associated with worse overall survival (OS).

Table 3. Results of liver transplantation for neuroendocrine tumors liver metastases (series of at least 10 patients, published in English after 1990).

\begin{tabular}{|c|c|c|c|c|c|}
\hline Study & Postoperative mortality & Overall survival & Disease-free survival & Survival prognostic factors & Ref. \\
\hline \multicolumn{6}{|c|}{ Multicentric series } \\
\hline Le Treut & $\begin{array}{l}\text { 14\%, risk factors: } \\
\text { - DP primary } \\
\text { - UAE } \\
\text { - Early re-LT }\end{array}$ & 5 years: $47 \%$ & 5 years: $20 \%$ & $\begin{array}{l}\text { UAE, DP primary, HMG. DP } \\
\text { primary }+\mathrm{HMG}=12 \% 5 \text { year OS }\end{array}$ & [11] \\
\hline Le Treut & $\begin{array}{l}\text { 10\%, risk factors: } \\
\text { - Early re-LT } \\
\text { - UAE } \\
\text { - Splenectomy } \\
\text { - Operative time >10 h } \\
\text { - R1-R2 resection } \\
\text { - HMG } \\
\text { - Concomitant surgery }\end{array}$ & 5 years: $52 \%$ & 5 years: $30 \%$ & $\begin{array}{l}\text { Major concomitant surgery, } \\
\text { poorly differentiated tumor, } \\
\text { HMG. After } 2000 \text { : HMG, age }>45 \text {, } \\
\text { concomitant resection }\end{array}$ & [9] \\
\hline Gedaly & & 5 years: $48 \%$ & 5 years: $32 \%$ & $\begin{array}{l}\text { Better survival if wait time }>67 \\
\text { days }\end{array}$ & [12] \\
\hline \multicolumn{6}{|c|}{ Monocentric series } \\
\hline Coppa & - & $\begin{array}{l}5 \text { years: } 67 \% \text { after } \\
L R, 70 \% \text { after } L T \\
(p=N S)\end{array}$ & $\begin{array}{l}5 \text { years: } 29 \% \text { after } L R, 53 \% \\
\text { after } L T(p=N S)\end{array}$ & - & {$[5]$} \\
\hline Van Vilsteren & $5 \%(n=1)$ & 1 year: 87\% & 1 year: $77 \%$ & $\begin{array}{l}\text { Ki67 correlated with survival, but } \\
\text { not with recurrence }\end{array}$ & [13] \\
\hline Rosenau & $5 \%(n=1)$ & 5 years: $80 \%$ & 5 years: $21 \%$ & $\begin{array}{l}\% \text { of Ki67-positive cells and } \\
\text { aberrant staining pattern for } \\
\text { E-cadherin- hepatichilar or } \\
\text { regional lymph node at LT }\end{array}$ & {$[8]$} \\
\hline Florman & $27 \%$ & 5 years: $36 \%$ & - & - & [16] \\
\hline Lang & $8 \%(n=1)$ & 5 years: $77 \%$ & - & - & {$[17]$} \\
\hline Lang & 0 & 5 years: $40 \%$ & 5 years: $10 \%$ & - & [18] \\
\hline Routley & 0 & 5 years: $57 \%$ & - & - & [19] \\
\hline Ahlman & $0 \%$ & - & - & - & [20] \\
\hline Personal series & $12 \%$ & 5 years: $55 \%$ & 5 years: $45 \%$ & - & \\
\hline
\end{tabular}


Eventually, some NET patients had lowgrade symptoms but were considered for LT in case of uncontrolled LMs, not amenable to liver resection $[9,27]$.

Noticeably, transarterial chemoembolization complications (sclerosing cholangitis and/or hepatic failure) indicated LT in 9/213 cases in the 2013 European series [9] and in $1 / 19$ cases in the German experience [8]. Table 2 summarizes indications for LT in the main published series.

Most of the patients had received previous surgical or medical treatment for LMs before LT. In Le Treut et al.'s series [9], 26\% of the patients had surgical procedures for LMs, including major hepatic resections in $11 \%$, and $76 \%$ of the patients received systemic therapies, including SSA in 30\% and TACE in 35\%.

In summary, current indications for LT are refractory hormonal syndrome and uncontrolled LMs, not amenable to other treatments.

What are the contraindications to LT?

High Ki67 index (>20\%), extrahepatic disease including unresectable distant lymph nodes metastases, poor performance status and severe carcinoid heart disease [23] represent the most prevalent contraindications to LT (see below).

Relative contraindications are unknown primary tumor, primary tumor without portal vein drainage, HMG, hepatic or hilar lymph node involvement, the necessity of upper abdominal exenteration (UAE) associated with multivisceral transplantation (MVT).

How should the staging of the disease be performed?

- Morphological assessment

As stated in the 2012 European Neuroendocrine Tumor Society (ENETS) guidelines [2], extrahepatic disease should be ruled out before LT is considered. At least 6 months before LT are required to both exclude tumors with an aggressive behavior and microscopic extrahepatic disease. In this setting, prior medical treatment of LMs allows a sufficient interval time to observe the natural history of the disease [28].

Thorough explorations should be performed [2]: SPECT/CT-somatostatin receptor (SMS-R), PET/CT using a ${ }^{68} \mathrm{Ga}-S S A$, and in special situations FDG-PET/CT (e.g., in NET G2) or alternative tracers $\left(5-\mathrm{HTP},{ }^{18} \mathrm{~F}\right.$-DOPA) may be required. NETs that are positive with FDGPET/CT and negative with SMS-R imaging exhibit a more aggressive profile [28] and might not represent good candidates for LT, although this issue has not been specifically addressed.

Staging laparoscopy or laparotomy $[13,29,30]$ can be performed before LT. It is not mandatory, especially if a comprehensive exploration of the abdominal cavity has been made during primary tumor removal.

\section{- Histological assessment}

Most of the series reported LT for well-differentiated NETs; patients with poorly differentiated tumors represented up to $10 \%$ in the European series [9]. Only four series with more than 10 cases reported the Ki67 index $[8,13,15,20]$.

Van Vilsteren et al. assessed $\mathrm{Ki} 67$ index in 18 explanted livers [13]. In $83 \%$ of the patients, Ki67 was less than 2\% (G1). Ki67 was higher in gastro-entero-pancreatic than midgut NETs, and in nonfunctional than functional tumors. Ki67 index was correlated with OS ( $\mathrm{p}=0.02)$.

Rosenau et al. studied Ki67 index, E-Cadherin and p53 expression in 19 explanted livers [8]. Survival was better $(\mathrm{p}=0.007)$ with low Ki67 and regular E-Cadherin pattern than with high Ki67 or loss of membranous E-Cadherin expression, with a $5 \%$ cut-off value for Ki67 index. The combination of $\mathrm{Ki} 67$ and E-Cadherin expression had specificity and sensitivity of $100 \%$ to predict survival 7 years after LT $(\mathrm{p}=0.003)$.

No consensus exists about Ki67 cut-off value as selection criteria for LT in NET patients. Based on the results of larger series and expert opinion [11,28], a Ki67 of $10 \%$ should not be exceeded.

Two factors should be taken into account when assessing Ki67 index: first, about half of the NETs metastatic to the liver show intratumoral heterogeneity resulting in discrepant Ki67 grade [31]; second, in some cases, a dedifferentiation of NETs can be observed with a much higher Ki67 index in metastasis than in the primary tumor [32]. Assessment of mitotic count and Ki67 index should therefore be performed both on the primary tumor pathological specimen and LMs, if possible at different locations.

What is the strategy with the primary tumor?

In the main published series (see Table 2), majority of primary tumors originates from the midgut (distal stomach, duodenum, small bowel, colon) and pancreas, which have portal venous drainage. In their review published in 
2007 [28], Mazzaferro et al. recommended that only metastatic NET patients with a primary tumor originating from the midgut should be candidates for LT. Their hypothesis was that the liver acts as a filter and 'first station' colonized during hematogenous spread of malignant cells, thus primary tumors without portal venous drainage might have more diffuse spreading.

However, location of the primary tumor did not appear to be a significant prognostic factor in the different studies reported in Table 2, except for Le Treut et al. that showed that a duodenopancreatic primary tumor negatively impacted OS, even when UAE was performed [11]. In the European series [9], duodenopancreatic primary site was only retrieved as a prognostic factor in univariate analysis.

Moreover, the primary tumor should be resected before LT to avoid major resections concomitant to LT. Concomitant resections of the primary tumor, especially if the latter requires a pancreatic resection, were shown to increase postoperative morbidity and have a negative impact on survival $[9,33,34]$.

The primary tumor resection should include a lymph node dissection, especially for NETs originating from the jejuno-ileum and appendix [35]; lymph node sampling only remaining inadequate. Furthermore, removal of primary tumor allows precise staging of the disease including assessment of lymph node status and vascular invasion, which are associated with poor OS [36].

In published studies, the primary site was unknown before LT in 5\% [8] up to $16 \%$ [11]. Nonetheless, these series extend over a long period and it can be assumed that now, with the development of SMS-R imaging and staging laparotomy, the percentage of unknown primary should decrease.

In the European series [9], unknown primary tumor was not a prognostic factor in univariate analysis. A 25-case European series from the same authors [37] reported 11 patients in whom the primary tumor was identified and treated during or after LT, and 14 patients whose primary tumor had never been identified. The actuarial OS rate of the unknown primary group was $55 \%$ at 5 years. There was no difference in terms of OS whether the primary site was subsequently identified or not, and there was no difference between patients who received LT without previously identification of the primary $(\mathrm{n}=25)$ and patients whose primary tumor was identified before LT $(n=150)$.
However, the identification and resection of the primary tumor before LT is advisable to decrease tumor load and avoid major resections concomitant to LT. Moreover, a minimal period of observation of 6-12 months after resection of primary tumor confirms the lack of disease progression before proposing LT [28].

What are the indications for UAE \& cluster transplantation?

In the beginning of the experience of LT for metastatic NETs, UAE and MVT in cluster were performed $[4,6,11,12,15,18,20,29,36,38]$.

The attitude toward UAE and MVT seems different between Europe and USA.

In the French multicenter study published in 2008 [11], UAE was associated with high postoperative mortality $(\mathrm{p}<0.001)$ and poor OS in multivariate analysis (relative risk $=3.7$, $\mathrm{p}=0.0034)$. These results were confirmed in the European series published in 2013 [9]: all 10 UAE were performed before 2000, and were associated with increased postoperative mortality. Similarly, major concomitant resection was found to be an independent prognostic factor for OS (hazard ratio: 3.1; $95 \mathrm{CI}$ : 1.9-5.3; $\mathrm{p}<0.0001)$. In the review published by Lehnert, UAE was associated with a poor prognosis [34]. Thus, UAE and MVT have been progressively abandoned in Europe after 2000 because of high postoperative mortality and poor OS results.

The Pittsburgh team [38] described their experience in MVT in a series from 1988 to 1992 and reported 21 cluster transplantations with only $25 \%$ of the LT indications for NETs. In contrast to the European experience, the incidence of the nontumor-related mortality was $33 \%$. Gedaly et al. further reported the results from the United Network for Organ Sharing with 13 MVT for NETs, with good results in terms of OS: 1- and 5-year survival were 80 and $48 \%$, respectively (vs 81 and $49 \%$, respectively, for LT only).

Recently, results from a multicenter database were reported by Sher et al. with a total of 17 MVT for metastatic NETs, six of which were performed before 2000 and 11 after 2000 [36]. OS rates were not different between MVT and LT only, with an increase in survival rates before and after 2000 for MVT. In this study, the 1and 3-year OS rates for MVT performed after 2000 were 88 and 44\%, respectively, versus 67 and $33 \%$ before 2000 . 
What other prognostic factors have been identified?

Extent of liver disease is not commonly reported in the literature; however, the team from Milan $[5,28]$ propose less than $50 \%$ liver metastatic involvement as a selection criterion for LT.

Age of the recipient is a prognostic factor in several studies $[9,33,34]$ with a threshold between 45 and 55 years.

Positive hepatic hilar lymph nodes at the time of LT were shown to be associated with impaired survival in two studies [8,9]; however, van Vilsteren et al. did not confirm this result [13]. Systematic pedicular lymphadenectomy during LT procedure can be performed for staging [28].

Is there a specific peri operative management regarding to the type of NET? A specific management of NET patients is required before and during surgical procedures. An exhaustive description has been made in the 2009 ENETS consensus guidelines for the standards of care in NETs [39].

In the preoperative period, patients with severe diarrhea, weight loss and hypoproteinemia may require parenteral nutrition. Patients with carcinoid syndrome must all have echocardiography, searching for carcinoid heart disease. They must as well receive SSA (octreotide) prophylaxis to prevent carcinoid crisis during surgery.

Concerning anesthesia, drugs that may stimulate sympathetic system or cause histamine release should be excluded. In case of hypotension, sympathomimetic drugs should be avoided, and intravenous octreotide should be given. In case of bronchospasm or flushing during anesthesia, octreotide administration should be reinforced, in addition to other therapies, with a progressive weaning during the first week. For patients with functional pancreatic NETs, specific measures should be undertaken according to the hormone secretion: proton pump inhibitors for gastrinoma, glucose monitoring for insulinoma, SSA treatment, nutritional supplementation and thrombosis prophylaxis for somatostatinoma and preoperative SSA treatment with intravenous fluid and electrolyte therapy for VIPoma.

Post-LT immunosuppression could be adapted with low-dose calcineurin inhibitors as soon as possible after LT and co-prescription of mTOR inhibitors. However, data are lacking to recommend a specific immunosuppression protocol.
What are the results in terms of operative mortality?

In large series ( $>15$ patients), post-LT mortality ranged from $5[8,13]$ up to $14 \%$ [11], with mortality rate improving over time. Risk factors that were identified were: UAE [9,11], early re-transplantation [9,11], duodenopancreatic primary [11], concomitant resection surgery, HMG, R1-R2 resection, operative time $>10 \mathrm{~h}$ and splenectomy [9].

\section{What are long-term results?}

As shown in Table 3, OS rates varied greatly between studies. Larger series reported 5-year survival rates around $50 \%[4,7,9,11,12]$. Two studies remarkably showed 70 [5] and $80 \%$ [8] 5-year survival rates. In the latter studies, strict selection criteria were applied for LT indication: all NETs were well-differentiated in Rosenau et al.'s study, and for Coppa et al., LT was proposed only to patients with age $<50$ years, primary carcinoid tumors from the gastrointestinal tract, stable disease without extrahepatic spread and liver involvement $<50 \%$.

Mazzaferro et al. recently compared patients with LMs from gastro-entero-pancreatic NETs who either received LT, nontransplant options, liver resection or medical treatment [40]. Patients were comparable for tumor burden, grading and stage. LT was proposed only if the primary was previously removed and with Ki67 $<10 \%$. Patients who received LT had a significant increased survival on multivariate analysis compared with those who did not (hazard ratio: 5.5; $\mathrm{p}=0.012$ ), with remarkable 5- and 10-year survival rates of 96.8 and $92.2 \%$, respectively. In our series (Tables $2 \& 3$ ), 5 -year OS rate was $55 \%$. However, this series reflects the beginning of our experience when selection criteria were not as strict as those proposed by Mazzaferro et al., in other words, with LT for unknown primary or primary tumor without portal venous drainage. However, randomized studies comparing outcomes of nonsurgical treatments and LT are not feasible, making the assessment of real benefit difficult [23]. A retrospective study showed that young patients with small intestine NETs reach 92\% 5-year OS rates with standardized multimodality treatment, questioning the role of LT in this particular setting [41].

Regarding hormonal secretion (e.g., carcinoid syndrome), LT offers a sustained relief, as LMs are far the most responsible for hormonal secretion-linked symptoms. However, 
data on completeness and duration of symptom relief after LT are poor. In our experience, we did not encounter major issues with endocrinopathies-related symptoms when disease recurred after LT.

Five-year recurrence-free survival rates ranged from 20 to $30 \%$ in largest series $[9,11,12]$. In Coppa et al.'s study comparing liver resection and LT; recurrence-free survival rates at 5 year were respectively 29 and $53 \%$ [5].

Is there a role for neoadjuvant or adjuvant treatment?

A downstaging strategy before LT has not been assessed in patients with NET LMs [23]. As LT is not the first-line option and is integrated into a multimodal treatment, bridging therapies such as TACE or SSA could be considered as neoadjuvant therapies. The impact of liver resection as a bridge to LT has not been assessed, yet in the European series [9], 11\% of patients underwent major liver resection before LT.

Adjuvant treatment is not recommended after liver resection $[23,42]$. Even if there is no specific trial, adjuvant treatment after LT does not seem justified. However, prescription of mTOR inhibitors as part of the post-transplant immunosuppressive regimen could be considered as an adjuvant treatment, despite reduced dosage.

\section{When should LT be proposed to metastatic NET patients?}

There is no evidence in the literature on the right timing to propose LT to these patients with a relatively slow evolution profile of the disease. Patients are mostly young and will be treated for numerous years, sometimes decades; hence the treatment strategy should take into account long-term side effects. In the authors' opinion, as other efficient strategies are available (SSA, TACE, systemic chemotherapy), the latter should be proposed in the first place. This attitude allows a period of observation of the disease, in order not to propose LT to patients with rapidly progressing LMs or early development of extrahepatic disease. On the other hand, the 'window' for LT should not be exceeded, potential risks being: a patient too 'old' to undergo LT,

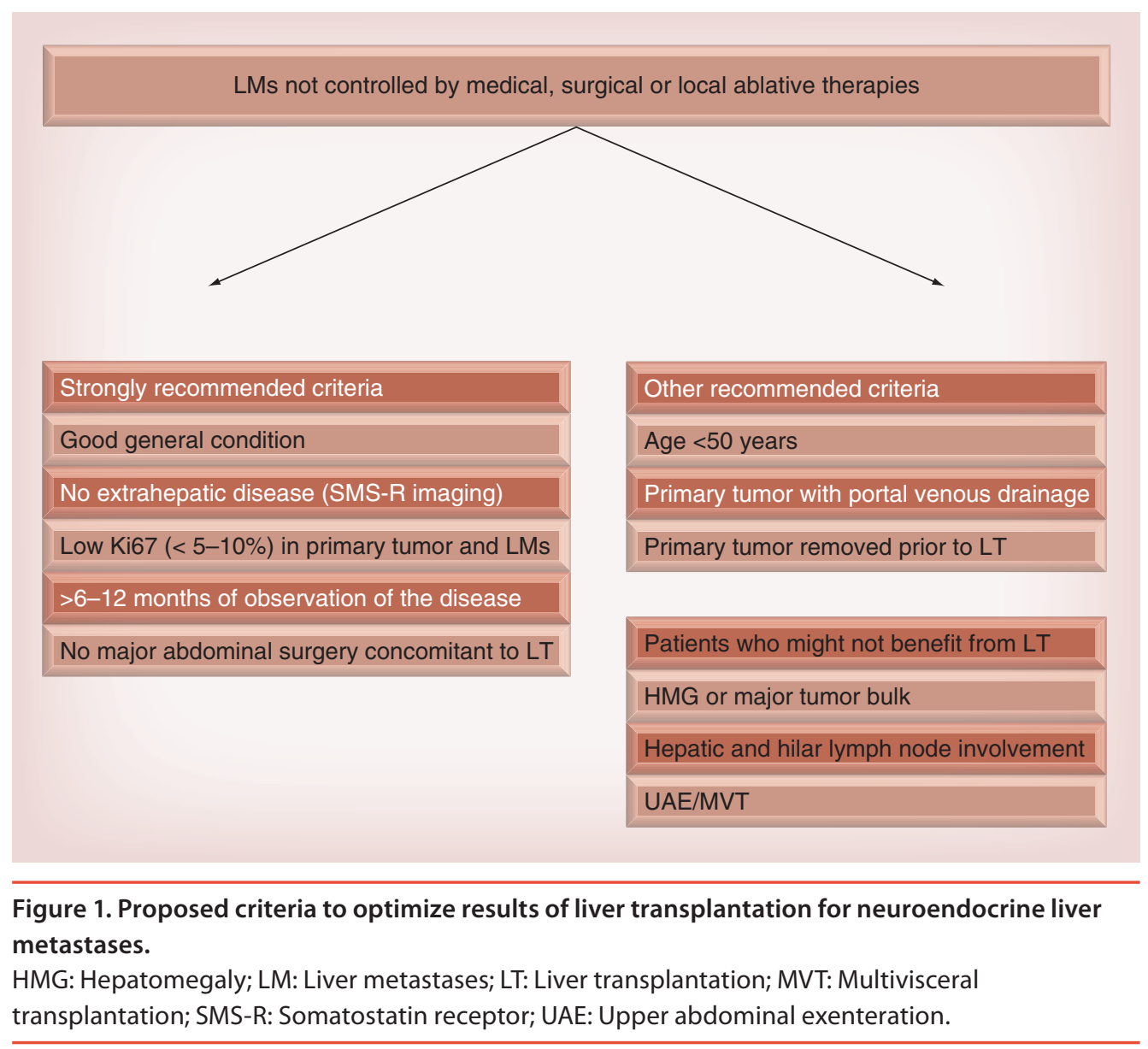


liver involvement progressing over $50 \%$ or late onset of extrahepatic disease, arterial complications of TACE compromising the success of LT and the development of a severe carcinoid heart disease contraindicating LT.

Patients with neuroendocrine LMs usually do not have underlying liver disease or liver insufficiency (with the notable exception of TACE complications) and therefore should benefit from a certain prioritization on the waiting list, depending on local allograft allocation conditions [43]. Living donor transplantation could as well become a viable option [23].

\section{Conclusion}

Indications of LT for NETs are not consensual, and the potential benefits should be weighed against peri operative morbidity and ethical distribution of donor organs. In light of this review, criteria could be proposed to improve short- and long-term results of LT for neuroendocrine LMs (Figure 1). Patients with good performance status, no competitive comorbidity (i.e., contraindication to surgery), no extrahepatic involvement, low Ki67 $(<5-10 \%)$ in primary tumor and LMs, primary tumor with portal venous drainage, stable disease for more than 6 months and without major abdominal surgery concomitant to LT might benefit the most from LT. It is preferable that primary tumor originates from the midgut and that it has been resected with an appropriate lymph node dissection before LT. If patients are strictly selected, and indicated for LT only after a long period of follow-up and when all other therapies appeared to be inefficient, 5 -year survival rates up to $75 \%$ may be observed.

\section{Future perspective}

Patients with good performance status, no competitive comorbidity (i.e., contraindication to surgery), no extrahepatic involvement, low Ki67 (<5-10\%) in primary tumor and LMs, primary tumor with portal venous drainage, stable disease for more than 6 months and without major abdominal surgery concomitant to LT might benefit the most from LT. With the more precise knowledge of survival prognostic factors and improvement in immunosuppression management, indications for LT in metastatic NET patients will be more consensual and restricted to patients whose expected OS rate at 5 years will reach $75 \%$.

Financial \& competing interests disclosure

The authors have no relevant affiliations or financial involvement with any organization or entity with a financial interest in or financial conflict with the subject matter or materials discussed in the manuscript. This includes employment, consultancies, honoraria, stock ownership or options, expert testimony, grants or patents received or pending or royalties.

No writing assistance was utilized in the production of this manuscript.

\section{References}

Papers of special note have been highlighted as:

- of interest; $\bullet$ of considerable interest

1 Yao JC, Hassan M, Phan A et al. One hundred years after "carcinoid": epidemiology of and prognostic factors for neuroendocrine tumors in 35,825 cases in the United States. J. Clin. Oncol. 26(18), 3063-3072 (2008).

2 Pavel M, Baudin E, Couvelard A et al. ENETS Consensus Guidelines for the management of patients with liver and other distant metastases from neuroendocrine neoplasms of foregut, midgut, hindgut, and unknown primary. Neuroendocrinology 95(2), 157-176 (2012).

- The European Neuroendocrine Tumor Society consensus guidelines on management of neuroendocrine liver metastases (LMs).

3 Adam R, Karam V, Delvart V et al. Frontiers in liver transplantation evolution of indications and results of liver transplantation in Europe. A report from the European Liver Transplant Registry. $J$. Hepatol. 57(3), 675-688 (2012).

4 Bechstein WO, Neuhaus P. Liver transplantation for hepatic metastases of neuroendocrine tumors. Ann. NY Acad. Sci. 733, 507-514 (1994).

5 Coppa J, Pulvirenti A, Schiavo M et al. Resection versus transplantation for liver metastases from neuroendocrine tumors. Transplant. Proc. 33(1-2), 1537-1539 (2001).

- Although this study has limited number of patients who received liver transplantation, this is the first study comparing resection and transplantation, with strict selection criteria for transplantation and promising results.

6 Le Treut YP, Delpero JR, Dousset B et al. Results of liver transplantation in the treatment of metastatic neuroendocrine tumors. A 31-case French multicentric report. Ann. Surg. 225(4), 355-364 (1997).

7 Nguyen NTT, Harring TR, Goss J, O’Mahony C. Neuroendocrine liver metastases and orthotopic liver transplantation: The US experience. Int. J. Hepatol. 2011, 742890 (2011).

8 Rosenau J, Bahr MJ, von Wasielewski R et al. Ki67, E-cadherin, and $\mathrm{p} 53$ as prognostic indicators of long-term outcome after liver transplantation for metastatic neuroendocrine tumors. Transplantation 73(3), 386-394 (2002).

-. The authors assessed Ki67 index and E-cadherin pattern expression in LMs and their impact on prognostic accuracy.

9 Le Treut YP, Grégoire E, Klempnauer J et al. Liver transplantation for neuroendocrine tumors in Europe-results and trends in patient selection: a 213-case European Liver 
Transplant Registry study. Ann. Surg. 257(5), 807-815 (2013).

- The updated multicentric European study with more than 200 patients included, and anaysis of survival prognostic factors.

10 Rindi G, Arnold R, Bosman FT et al. Nomenclature and classification of neuroendocrine neoplasms of the digestive system. In: WHO Classification of Tumours of the Digestive System (4th Edition). Bosman FT, Carneiro F, Hruban RH, Theise ND (Eds). IARC, Lyon, France, 13-14 (2010).

11 Le Treut YP, Grégoire E, Belghiti J et al. Predictors of long-term survival after liver transplantation for metastatic endocrine tumors: an 85-case French multicentric report. Am. J. Transplant. 8(6), 1205-1213 (2008).

- The first multicentric European study with univariate and multivariate analysis of survival prognostic factors.

12 Gedaly R, Daily MF, Davenport D et al. Liver transplantation for the treatment of liver metastases from neuroendocrine tumors. Arch. Surg. 146(8), 953-958 (2011).

13 Van Vilsteren FGI, Baskin-bey ES, Nagorney $\mathrm{DM}$ et al. Liver transplantation for gastroenteropancreatic neuroendocrine cancers?: defining selection criteria to improve survival. Liver Transpl. 59427, 448-456 (2006).

- Focuses on Ki67 index in explanted livers and the impact on survival and recurrence.

14 Marín C, Robles R, Fernández JA et al. Role of liver transplantation in the management of unresectable neuroendocrine liver metastases. Transplant. Proc. 39(7), 2302-2303 (2007).

15 Cahlin C, Friman S, Ahlman H et al. Liver transplantation for metastatic neuroendocrine tumor disease. Transplant. Proc. 35(2), 809-810 (2003).

16 Florman S, Toure B, Kim L et al. Liver transplantation for neuroendocrine tumors. J. Gastrointest. Surg. 8(2), 208-212 (2004).

17 Lang H, Oldhafer KJ, Weimann A et al. Liver transplantation for metastatic neuroendocrine tumors. Ann. Surg. 225(4), 347-354 (1997).

18 Lang H, Schlitt HJ, Schmidt H et al. Total hepatectomy and liver transplantation for metastatic neuroendocrine tumors of the pancreas -- a single center experience with ten patients. Langenbeck's Arch. Surg. 384(4), 370-377 (1999).

19 Routley D, Ramage JK, McPeake J, Tan KC, Williams R. Orthotopic liver transplantation in the treatment of metastatic neuroendocrine tumors of the liver. Liver Transpl. Surg. 1(2), 118-121 (1995).
20 Ahlman H, Friman S, Cahlin C et al. Liver transplantation for treatment of metastatic neuroendocrine tumors. Ann. NY Acad. Sci. 1014(1), 265-269 (2004).

21 Klimstra DS, Modlin IR, Coppola D, Lloyd RV, Suster S. The pathologic classification of neuroendocrine tumors a review of nomenclature, grading, and staging systems. Pancreas 39 (6), 707-712 (2010).

22 Jensen RT, Cadiot G, Brandi ML et al. ENETS Consensus Guidelines for the management of patients with digestive neuroendocrine neoplasms: functional pancreatic endocrine tumor syndromes. Neuroendocrinology 95(2), 98-119 (2012).

23 Frilling A, Modlin IM, Kidd M et al. Working Group on Neuroendocrine Liver Metastases. Recommandations for management of patients with neuroendocrine liver metastases. Lancet Oncol. 15(1), e8-e21 (2014).

-. Up-to-date recommandations for management of patients with neuroendocrine LMs.

24 Mayo SC, Herman JM, Cosgrove D et al. Emerging approaches in the management of patients with neuroendocrine liver metastasis: role of liver-directed and systemic therapies. J. Am. Coll. Surg. 216(1), 123-134 (2013).

25 Fteriche FA, Dokmak S, Aussilhou B et al. Liver resection for neuroendocrine liver metastases gives excellent long term survival but high recurrence rate. Program and Abstracts of the 10th Congress Of European-African Hepato Pancreato Biliary Association. Belgrade, Serbia, 29-31 May 2013. (Abstract no.743837)

26 Eriksson B, Annibale B, Bajetta E et al. ENETS Consensus Guidelines for the standards of care in neuroendocrine tumors: chemotherapy in patients with neuroendocrine tumors. Neuroendocrinology 90(2), 214-219 (2009).

27 Dousset B, Houssin D, Soubrane O, Boillot O, Baudin F, Chapuis Y. Metastatic endocrine tumors: is there a place for liver transplantation? Liver Transpl. Surg. 1(2), 111-117 (1995).

28 Mazzaferro V, Pulvirenti A, Coppa J. Neuroendocrine tumors metastatic to the liver: how to select patients for liver transplantation? J. Hepatol. 47(4), 460-466 (2007).

-• The authors of this review discuss good indications for liver transplantation in metastatic neuroendocrine tumor patients to improve survival and decrease recurrence.
29 Olausson M, Friman S, Cahlin Cet al. Indications and results of liver transplantation in patients with neuroendocrine tumors. World J. Surg. 26(8), 998-100 (2002).

30 Pascher A, Klupp J, Neuhaus P. Endocrine tumours of the gastrointestinal tract. Transplantation in the management of metastatic endocrine tumours. Best Pract. Res. Clin. Gastroenterol. 19(4), 637-648 (2005).

31 Yang Z, Tang LH, Klimstra DS. Effect of tumor heterogeneity on the assessment of Ki67 labeling index in well-differentiated neuroendocrine tumors metastatic to the liver: implications for prognostic stratification. Am. J. Surg. Pathol. 35(6), 853-860 (2011).

32 Poian C, Neam u MC, Avramescu ET et al. The dedifferentiation of neuroendocrine tumor metastases: myth or reality? Rom. J. Morphol. Embryol. 54(1), 201-203 (2013).

33 Máthé Z, Tagkalos E, Paul A et al. Liver transplantation for hepatic metastases of neuroendocrine pancreatic tumors: a survival-based analysis. Transplantation 91(5), 575-582 (2011).

34 Lehnert T. Liver transplantation for metastatic neuroendocrine carcinoma: an analysis of 103 patients. Transplantation 66(10), 1307-1312 (1998).

35 Pape UF, Perren A, Niederle B et al. ENETS Consensus Guidelines for the management of patients with neuroendocrine neoplasms from the jejuno-ileum and the appendix including goblet cell carcinomas. Neuroendocrinology 95(2), 135-156 (2012).

36 Sher L, Petrovic LM, Schwartz M et al. Abstracts of the ILTS (International Liver Transplantation Society) 19th Annual International Congress. June 11-14, 2013. Liver Transpl. 19(Suppl. 1), S86-S334 (2013).

37 Grégoire E, Le Treut YP, Klempnauer J. Oral abstracts of the American Transplant Congress 2010. Am. J. Transplant. 10, 37-212 (2010).

38 Alessiani M, Tzakis A, Todo $S$ et al. Assessment of five-year experience with abdominal organ cluster transplantation. J. Am. Coll. Surg. 180(1), 1-9 (1995).

39 Akerström G, Falconi M, Kianmanesh R, Ruszniewski P, Plöckinger U. ENETS consensus guidelines for the standards of care in neuroendocrine tumors: pre- and perioperative therapy in patients with neuroendocrine tumors. Neuroendocrinology 90 (2), 203-208 (2009). 
40 Mazzaferro V, Sposoto C, Miceli R et al. Magnitude of survival benefit after liver transplantation for metastatic neuroendocrine tumors of gastro-entero-pancreatic origin. Presented at: AASLD, The Liver Meeting. Washington DC, USA, 1-5 November 2013.

41 Norlen O, Daskalakis K, Öberg K, Akerstrom G, Stalberg P, Hellman P. Indication for liver transplantation in young patients with slall intestinal NETs is rare? World J. Surg. 38, 742-747 (2014).

42 Maire F, Hammel P, Kianmanesh R et al. Is adjuvant therapy with streptozotocin and 5-fluorouracil useful after resection of liver metastases from digestive endocrine tumors? Surgery 145(1), 69-75 (2009).
43 Francoz C, Belghiti J, Castaing D et al. Model for end-stage liver disease exceptions in the context of the French model for end-stage liver disease score-based liver allocation system. Liver Transpl. 17(10), 1137-1151 (2011). 